\title{
NEUTRINO SPIN-FLAVOR OSCILLATIONS IN RAPIDLY VARYING MAGNETIC FIELDS
}

\author{
M.Dvornikov ${ }^{a}$ \\ Department of Physics, University of Jyväskylä, Finland and \\ IZMIRAN, Troitsk, Russia
}

\begin{abstract}
The general formalism for the description of neutrino oscillations in arbitrary rapidly varying external fields is elaborated. We obtain the new effective Hamiltonian which determines the evolution of the averaged neutrino wave function. The general technique is applied to the neutrino oscillations in rapidly varying magnetic fields. We evaluate the transition probabilities of the neutrino spin-flavor oscillations in magnetic fields of the Sun and compare them with the numerical solutions of the Schrödinger equation with the exact Hamiltonian.
\end{abstract}

One of the most interesting problems in the neutrino physics is the solar neutrino deficit. Nowadays it is experimentally established that the disappearance of solar electron neutrinos can be accounted for by the LMA-MSW solution. Apart from the LMA-MSW solution other theoretical models of the solar neutrino oscillations like spin-flavor precession (see work [1] and references therein) are also considered. We mention that neutrino oscillations in electromagnetic fields of various configurations were examined in our previous works (see Refs. [2]).

In this paper we study neutrino oscillations in presence of general rapidly varying fields. We derive the new effective Hamiltonian for the time evolution of the averaged neutrino wave function. Then we apply the general technique to neutrino oscillations in rapidly varying magnetic fields and discuss the neutrino conversion in solar magnetic fields. We present the numerical solutions of Schrödinger equation for the neutrino system interacting with the constant transversal and twisting magnetic fields and compare them with the approximate analytical solutions found in this work.

Let us consider the evolution of the two neutrinos $\nu=\left(\nu_{1}, \nu_{2}\right)$, which can belong to different flavors and helicity states. The evolution of the system is described by the Schrödinger type differential equation,

$$
i \frac{d \nu}{d t}=H \nu
$$

Here we do not specify the explicit form of the Hamiltonian but just suppose that it is decomposed into two terms,

$$
H=H_{0}+\mathcal{H}, \quad \mathcal{H}(t+T)=\mathcal{H}(t) .
$$

The Hamiltonian $H_{0}$ corresponds to the neutrino interaction with constant or slowly varying external fields. In presence of only this term the solution of

${ }^{a}$ e-mail: maxim.dvornikov@phys.jyu.fi 
Eq. (1) can be easily found. It is known to be periodical with the typical frequency $\Omega_{0} \sim 1 / L_{\text {eff }}$, where $L_{\text {eff }}$ is the oscillations length. The function $\mathcal{H}(t)$ corresponds to rapidly varying external fields. The frequency $\omega=2 \pi / T$ should be much greater than $\Omega_{0}$.

We will seek the solution of Eqs. (1) and (2) in the form (see also Ref. [3]),

$$
\nu(t)=\nu_{0}(t)+\xi(t) .
$$

In Eq. (3) the function $\xi(t)$ is the small rapidly oscillating one with zero mean value and the function $\nu_{0}(t)$ is the slowly varying one during $T$. The mean value of a function is the time averaging over the period $T$. After substituting Eq. (3) in Eq. (1) we obtain two groups of rapidly and slowly varying terms in Eq. (1). In order for this expression to be an identity the terms of each group in left-handed side of the equation should be equal to the terms of the same group in right-handed side. With help of this fact one finds the time dependence of $\xi$ in the explicit form,

$$
\xi(t)=-i\left(\int \mathcal{H}(t) \mathrm{d} t\right) \nu_{0}(t) .
$$

Averaging residual terms in Eq. (1) over the period $T$ and accounting for Eq. (4) we obtain the equation for the function $\nu_{0}$,

$$
i \frac{d \nu_{0}}{d t}=H_{\mathrm{eff}} \nu_{0}, \quad H_{\mathrm{eff}}=H_{0}-i \overline{\mathcal{H}\left(\int \mathcal{H} \mathrm{d} t\right)} .
$$

In derivation of Eq. (5) we do not make any assumptions about the smallness of the interaction described by the Hamiltonian $\mathcal{H}$.

On the basis of the general technique we discuss the neutrino evolution in matter under the influence of a combination of the constant transversal $\mathbf{B}_{0}$ and twisting $\mathbf{B}(\mathbf{r})$ magnetic fields. In our case the Hamiltonians $H_{0}$ and $\mathcal{H}$ have the form

$$
H_{0}=\left(\begin{array}{cc}
V / 2 & \mu B_{0} \\
\mu B_{0} & -V / 2
\end{array}\right), \quad \mathcal{H}=\left(\begin{array}{cc}
0 & \mu B e^{-i \omega t} \\
\mu B e^{i \omega t} & 0
\end{array}\right)
$$

where $\mu$ is the neutrino magnetic moment. In Eq. (6) we introduce the quantity $V / 2=\left(\Delta m^{2} / 4 E\right) \Theta-G_{F} n_{\text {eff }} / \sqrt{2}$, where $\Theta$ is the function of the vacuum mixing angle $\theta_{\text {vac }}$ (see Ref. [4]), $\Delta m^{2}$ is the difference of the neutrino mass squared, $E$ is the neutrino energy, $n_{\text {eff }}$ is the effective matter density, $\omega$ is the frequency of the transversal magnetic field variation, $G_{F}$ is the Fermi constant.

Using Eqs. (5)-(6) it is possible to derive the expression for $H_{\text {eff }}$,

$$
H_{\text {eff }}=\left(\begin{array}{cc}
V / 2-(\mu B)^{2} / \omega & \mu B_{0} \\
\mu B_{0} & -V / 2+(\mu B)^{2} / \omega
\end{array}\right) .
$$


Since $B_{0}$ and $B$ do not depend on time in Eq. (7), we can solve Eq. (5) for the effective Hamiltonian given in Eq. (7). The transition probability is expressed in the following way

$$
P(t)=A \sin ^{2}\left(\frac{\pi t}{L}\right)
$$

where

$$
A=\frac{\left(\mu B_{0}\right)^{2}}{\left[V / 2-(\mu B)^{2} / \omega\right]^{2}+\left(\mu B_{0}\right)^{2}}, \quad \frac{\pi}{L}=\sqrt{\left[V / 2-(\mu B)^{2} / \omega\right]^{2}+\left(\mu B_{0}\right)^{2}} .
$$

As it results from Eqs. (8) and (9), if the following condition is satisfied,

$$
\frac{V}{2} \simeq \frac{(\mu B)^{2}}{\omega}
$$

then $A \simeq 1$ and the transition probability can achieve great values. This phenomenon is analogous to resonance amplification of spin-flavor oscillations.

It is interesting to discuss the situation when $B \gg B_{0}$. In this case the value of $A_{0}=A(B=0)$ is much less than unity. Hence the transition probability at the absence of the additional twisting magnetic field is small. In this case, if we choose the parameters according to Eq. (10), the amplitude of the transition probability becomes great. From Eqs. (9) and (10) one can derive the restriction imposed on $B$ and $B_{0},\left(B / B_{0}\right)^{2} \gg 1$.

Now we consider one of the possible applications of the developed technique to neutrino spin-flavor oscillations in magnetic fields of the Sun. The solar magnetic field is unlikely to be only either constant transversal or twisting. Therefore we can apply the method elaborated in this paper to the description of the solar neutrino conversion. We examine one of the possible channels of neutrino oscillations, namely $\nu_{e L} \leftrightarrow \nu_{\mu R}$ conversion. First one should estimate the parameter $V$ in Eq. (6). In our case $\Theta=\left(1+\cos 2 \theta_{\text {vac }}\right) / 2$ and the effective matter density $n_{\text {eff }}=\left(n_{e}-n_{n} / 2\right)$. Let us discuss the neutrino with the following properties, $\Delta m^{2} \sim 10^{-5} \mathrm{eV}^{2}, \theta_{\text {vac }} \sim \pi / 4$. We take the neutrino energy $E \sim$ $10 \mathrm{MeV}$. Matter is supposed to consist mainly of hydrogen and has the density $d \sim 1.4 \mathrm{~g} / \mathrm{cm}^{3}$. For these parameters we obtain that $V / 2 \sim 10^{-15} \mathrm{eV}$.

We can evaluate the strength of the magnetic fields necessary for the $10 \%$ conversion of the initial $\nu_{e L}$ beam. We assume that neutrino conversion occurs along the distance $D \simeq 3.2 \times 10^{14} \mathrm{eV}^{-1} \sim R_{\odot} / 10$, where $R_{\odot}$ is the solar radius. Neutrinos are supposed to have the transitional magnetic moment $\mu=10^{-11} \mu_{\mathrm{B}}$. Setting $P(t)=0.1$ in Eq. (8) we obtain $B_{0} \simeq 17.6 \mathrm{kG}$. Then we can derive the strength of the twisting magnetic field: $B \simeq 3.2 B_{0} \simeq 56.3 \mathrm{kG}$. The resonance condition (10) is satisfied if $\omega \simeq 1.0 \times 10^{-14} \mathrm{eV}$.

In order to substantiate the correctness of the approach developed in our paper we obtain the numerical solutions of Eq. (1) with the Hamiltonian given 


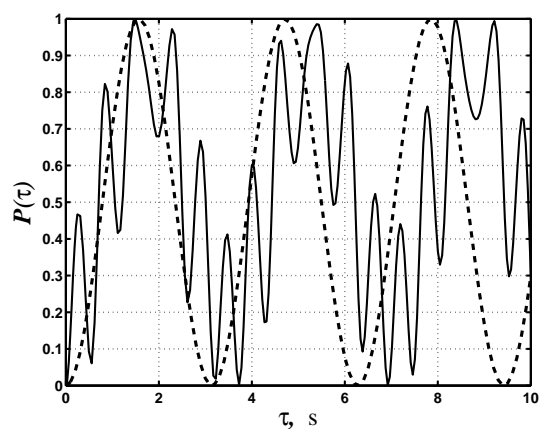

(a)

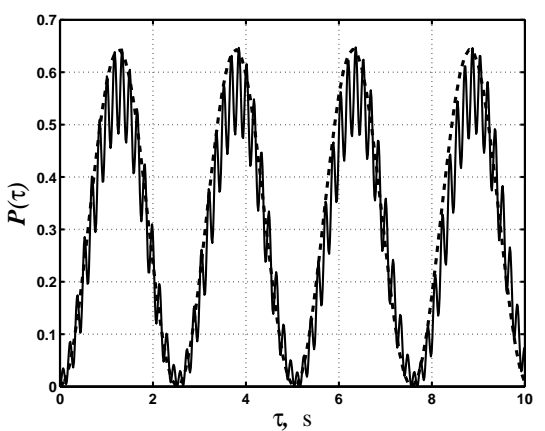

(b)

Figure 1: Neutrino transition probability for $\nu_{e L} \leftrightarrow \nu_{\mu}$ oscillations; (a) $\omega=1.0 \times 10^{-14} \mathrm{eV}$; (b) $\omega=4.0 \times 10^{-14} \mathrm{eV}$.

in Eq. (6). The probabilities of neutrino oscillations are shown on Fig. 1 versus $\tau=1.5 t$. We also compare them with the approximate analytical formula (8). The solid line corresponds to the transition probability computed with help of the numerical solution of Eq. (1) for the parameters taken above. The dashed line represents the transition probability given in Eq. (8). It can be seen in Fig. 1 that the numerical expression for the transition probability approaches to the approximate formula given in Eq. (8) at great frequencies of the twisting magnetic field. This comparison proves the validity of the elaborated technique.

\section{Acknowledgments}

This research was supported by the Academy of Finland under the contract No. 108875 and by a grant of Russian Science Support Foundation. The author is indebted to the organizers of the $12^{\text {th }}$ Lomonosov Conference on Elementary Particle Physics for inviting him to participate in this activity.

\section{References}

[1] E.Kh.Akhmedov, et al., Phys.Rev. D 48, 2167 (1993).

[2] M.S.Dvornikov, A.I.Studenikin, Phys.At.Nucl. 64, 1624 (2001); 67, 719 (2004).

[3] P.L.Kapitza, Usp.Fiz.Nauk 44, 7 (1951).

[4] G.G.Likhachev, A.I.Studenikin, JETP 81, 419 (1995). 